\begin{abstract}
Aimo Kannt
1991-1995 Studium der Biochemie in Tübingen und Cambridge (UK); 1995-1999 Promotion am MaxPlanck-Institut für Biophysik und der Universität Frankfurt a. M.; 1999-2001 Wissenschaftlicher Mitarbeiter am MPI für Biophysik; seit 2001 Pharmaforschung bei Sanofi, derzeit Abteilungsleiter, Begleitund Folgeerkrankungen des Diabetes; März 2017 Habilitation für das Fach Experimentelle Pharmakologie an der Universität Heidelberg.
\end{abstract}

DOI: $10.1007 / \mathrm{s} 12268-017-0861-\mathrm{x}$ (C) Springer-Verlag 2017

Als Konsequenz veränderter Ernährungsund Lebensgewohnheiten nimmt die weltweite Häufigkeit von kardiovaskulären und metabolischen Erkrankungen weiter zu. Trotz deutlicher Verbesserungen der medizinischen Versorgung und der verfügbaren Therapieoptionen sind Herz-Kreislauf-Erkrankungen noch immer Todesursache Nummer 1. Die Prävalenz des Typ-2-Diabetes ist nicht nur in der westlichen Welt sehr hoch, sondern besonders auch in Asien und Lateinamerika, mit entsprechenden Auswirkungen auf die dortigen Gesundheitssysteme. Es gibt einen deutlichen Bedarf für mehr und bessere Wirkstoffe für die Behandlung der Adipositas, des Diabetes und seiner Begleiterkrankungen und Komplikationen.

Gleichzeitig wird es immer schwieriger, neue Arzneimittel mit einem - im Vergleich zur Standardtherapie - Zusatznutzen für Patienten, Ärzte und Gesundheitssysteme zu entwickeln. Trotz enormer technologischer

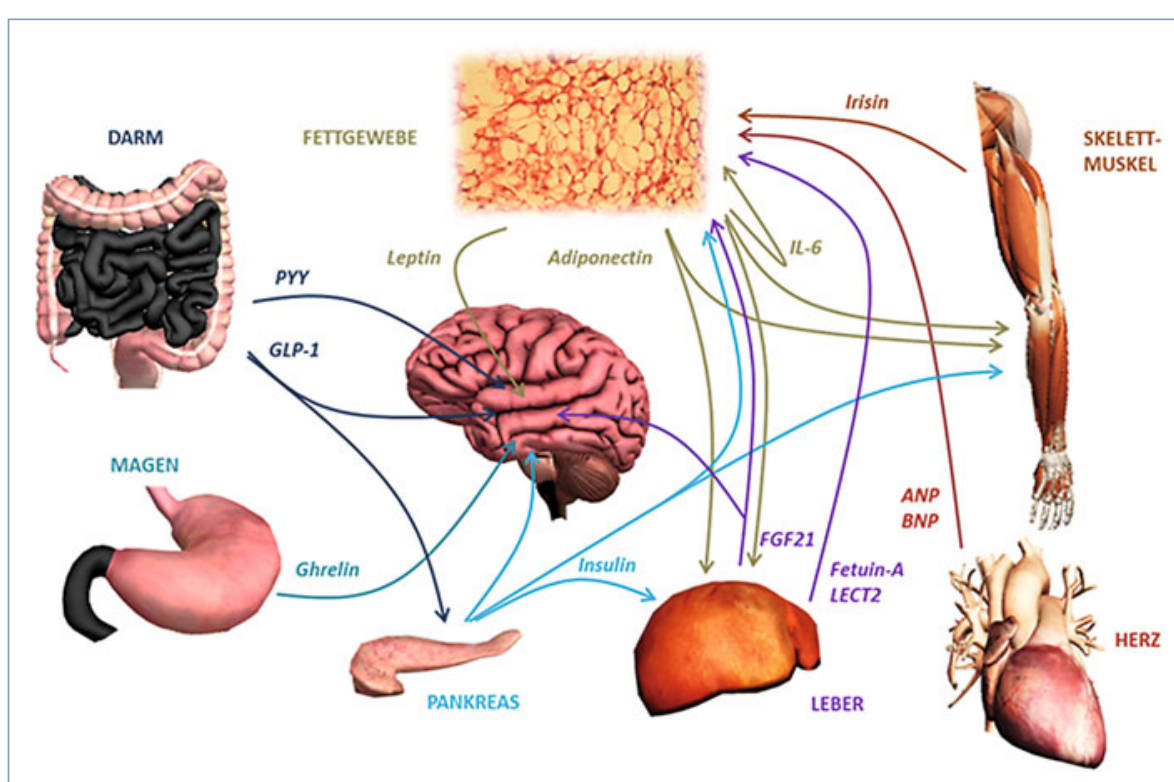

$\Delta$ Abb. 1: Hormonelle Regelkreise der Glukose- und Energiehomöostase. Die von den verschiedenen Geweben sezernierten Faktoren werden auch als Organokine bezeichnet. Einige dieser Organokine werden als neuartige Therapeutika für kardiometabolische Erkrankungen erforscht und klinisch geprüft. Abbildung aus [1], erstellt mit Zygote Body ${ }^{\top M}$.

\section{Habilitierte stellen sich vor}

\author{
AIMO KANNT \\ SANOFI DIABETES RESEARCH, FRANKFURT A. M.
}

und wissenschaftlicher Fortschritte ist die Produktivität der pharmazeutischen Forschung und Entwicklung (F\&E), gemessen an der Zahl neu zugelassener Medikamente im Verhältnis zu den F\&E-Kosten, in den letzten Jahrzehnten stetig gesunken. Die Gründe für diese Produktivitätsabnahme sind vielgestaltig und reichen von verbesserter und somit schwerer zu übertreffender Standardtherapie über höhere regulatorische Anforderungen an Arzneimittelwirksamkeit und -sicherheit bis hin zu ungenügendem Management von technischen und translationalen Risiken entlang der pharmazeutischen F\&E-Wertschöpfungskette.

In der Habilitationsschrift werden diese Risiken und deren Vermeidung oder Minimierung genauer beleuchtet. Ein spezieller Schwerpunkt ist dabei ein Risiko, das bislang nicht umfangreich gewürdigt und systematisch analysiert wurde - die mangelnde Reproduzierbarkeit publizierter Arbeiten. Anhand von Beispielen aus der eigenen Forschungsarbeit werden Wege aufgezeigt, um bestimm-

\title{
Wirkstofffindung in kardiovaskulären und metabolischen Erkrankungen
}

ten technischen und translationalen Schwierigkeiten $\mathrm{zu}$ begegnen und Wirkstofffindungsprogramme in kardiovaskulären und metabolischen Erkrankungen zur klinischen Reife zu bringen. Diese Beispiele umfassen die Übertragung von Befunden aus Tiermodellen auf Patienten mit Adipositas und Typ-2-Diabetes, die Identifizierung und Profilierung neuartiger anti-arrhythmischer Substanzen, die Devalidierung eines Ansatzes zur Behandlung der Adipositas sowie die Entwicklung von Testsystemen für die Wirkstofffindung. Die beschriebenen Arbeiten beschäftigen sich mit Enzymen und Rezeptoren, die in unterschiedlichen Regelkreisen - etwa dem Glukosestoffwechsel oder der kardialen Anregungs-Kontraktions-Kopplung und verschiedenen Zielorganen aktiv sind und damit ein breites Spektrum an möglichen pharmazeutischen Angriffspunkten für kardiovaskuläre und metabolische Erkrankungen abdecken.

Beispiele für solche metabolischen Regelkreise zur Glukose- und Energiehomöostase unter Beteiligung verschiedener Organe und der von ihnen sezernierten Faktoren sind in der Abbildung 1 dargestellt.

\section{Danksagung}

Mein besonderer Dank gilt Herrn Prof. Dr. Thomas Wieland und Herrn Prof. Dr. HansPeter Hammes für die Unterstützung meiner Habilitation. Darüber hinaus bedanke ich mich bei meinen früheren und jetzigen Kolleginnen und Kollegen, Mitarbeiterinnen und Mitarbeitern für die angenehme und erfolgreiche Zusammenarbeit.

\section{Literatur}

[1] Kannt A, Blüher M. 2015. Neue Therapieansätze zur Behandlung der Adipositas. Forschung Medizin Exzellenzforschung in der Medizin 5: 60-68

Korrespondenzadresse: PD Dr. Aimo Kannt Sanofi Diabetes Research Industriepark Hoechst, H823 D-65926 Frankfurt a. M. Tel.: 069-305-16351 aimo.kannt@sanofi.com 